\title{
RATIONALITY OF TWISTS OF THE SIEGEL MODULAR VARIETY OF GENUS 2 AND LEVEL 3
}

\author{
FRANK CALEGARI AND SHIVA CHIDAMBARAM
}

\begin{abstract}
Let $\bar{\rho}: G_{\mathbf{Q}} \rightarrow \operatorname{GSp}_{4}\left(\mathbf{F}_{3}\right)$ be a continuous Galois representation with cyclotomic similitude character. Equivalently, consider $\bar{\rho}$ to be the Galois representation associated to the 3-torsion of a principally polarized abelian surface $A / \mathbf{Q}$. We prove that the moduli space $\mathcal{A}_{2}(\bar{\rho})$ of principally polarized abelian surfaces $B / \mathbf{Q}$ admitting a symplectic isomorphism $B[3] \simeq \bar{\rho}$ of Galois representations is never rational over $\mathbf{Q}$ when $\bar{\rho}$ is surjective, even though it is both rational over $\mathbf{C}$ and unirational over $\mathbf{Q}$ via a map of degree 6 .
\end{abstract}

\section{INTRODUCTION}

Let $p$ be a prime and suppose that $A / \mathbf{Q}$ is an abelian variety of dimension $g$ with a polarization of degree prime to $p$. Associated to the action of the absolute Galois group $G_{\mathbf{Q}}$ on $A[p]$ there exists a Galois representation

$$
\bar{\rho}: G_{\mathbf{Q}} \rightarrow \operatorname{GSp}_{2 g}\left(\mathbf{F}_{p}\right)
$$

such that the corresponding similitude character is the mod- $p$ cyclotomic character $\varepsilon$. One can ask, conversely, whether any such representation comes from an abelian variety in infinitely many ways. When $g=1$, this question is well-studied, and has a positive answer exactly for $p=2,3$, and 5 . Indeed, the corresponding twists $X(\bar{\rho})$ of the modular curve $X(p)$ are rational over $\mathbf{Q}$ for $p=2,3$, and 5 , and have higher genus for larger $p$.

In BCGP18, this question arose for abelian surfaces $(g=2)$ when $p=3$. (The case $p=2$, which is also discussed in that paper, is understood by analyzing the branch points of the hyperelliptic involution.) Let $\mathcal{A}_{2}(3)$ denote the Siegel modular variety of genus 2 and level 3 . It is the moduli space of principally polarized abelian surfaces together with a symplectic isomorphism $A[3] \simeq(\mathbf{Z} / 3 \mathbf{Z})^{2} \oplus\left(\mu_{3}\right)^{2}$. Given a $\bar{\rho}$ as above, one can form the corresponding moduli space $\mathcal{A}_{2}(\bar{\rho})$ where now one insists that there is a symplectic isomorphism $A[3] \simeq V$, where $V$ is the representation space of $\bar{\rho}$ with its symplectic structure. The variety $\mathcal{A}_{2}(3)$ is well-known to be birational to the Burkhardt quartic, which is rational over $\mathbf{Q}$ (BN18]). It is clear that $\mathcal{A}_{2}(\bar{\rho})$ is isomorphic to $\mathcal{A}_{2}(3)$ over $\mathbf{C}$ (and even over the fixed field of the kernel of $\bar{\rho})$, and hence $\mathcal{A}_{2}(\bar{\rho})$ is geometrically rational. If $\mathcal{A}_{2}(\bar{\rho})$ was in fact rational (by which we always mean rational over the base field), then indeed the answer to the question above would be positive, just as for elliptic curves when $p \leq 5$. In BCGP18, Prop 10.2.3], a weaker result was established: The variety $\mathcal{A}_{2}(\bar{\rho})$ is unirational over $\mathbf{Q}$ via a map of degree at most 6 . As a consequence, any such $\bar{\rho}$ does arise from (infinitely many) abelian surfaces. We refer the reader to [CCR20]

Both authors were supported in part by NSF Grants DMS-1701703. The first author was supported in part by DMS-2001097, and the second author was supported in part by the Simons Foundation (grant 550033). 
which produces explicit polynomials describing the universal family over a rational cover of $\mathcal{A}_{2}(\bar{\rho})$ of degree 6 . However, the question as to whether $\mathcal{A}_{2}(\bar{\rho})$ was actually rational was left open. We address this question here.

Theorem 1. Let $\bar{\rho}: G_{\mathbf{Q}} \rightarrow \operatorname{GSp}_{4}\left(\mathbf{F}_{3}\right)$ be a representation with cyclotomic similitude character. Suppose that the order of $\operatorname{im}(\bar{\rho})$ is greater than 96 . Then $\mathcal{A}_{2}(\bar{\rho})$ is not rational over $\mathbf{Q}$.

More refined results can be extracted directly from the table in $\$ 3$ Since $\bar{\rho}$ has cyclotomic similitude character, the restriction of $\bar{\rho}$ to $G_{E}$, where $E=\mathbf{Q}(\sqrt{-3})$, has image contained in $\operatorname{Sp}_{4}\left(\mathbf{F}_{3}\right)$. If we let $H$ denote the projection of $\operatorname{im}\left(\left.\bar{\rho}\right|_{G_{E}}\right)$ to the simple group $\operatorname{PSp}_{4}\left(\mathbf{F}_{3}\right)$, then we prove that $\mathcal{A}_{2}(\bar{\rho})$ is not rational over $\mathbf{Q}$ for all but 26 of the 116 conjugacy classes of subgroups of $\operatorname{PSp}_{4}\left(\mathbf{F}_{3}\right)$. With the exception of three cases (including when $H$ is trivial) where the methods of [BN18] may be applied (see $\$ 2.3$ ), we do not know what happens in the remaining 23 cases, nor do we even know whether the rationality of $\mathcal{A}_{2}(\bar{\rho})$ depends only on $\operatorname{im}(\bar{\rho})$ or not. One easy remark is that, for a quadratic character $\chi$, there is an isomorphism $\mathcal{A}_{2}(\bar{\rho}) \simeq$ $\mathcal{A}_{2}(\bar{\rho} \otimes \chi)$, and so the rationality of $\mathcal{A}_{2}(\bar{\rho})$ depends only on the image of $\left.\bar{\rho}\right|_{G_{E}}$ in $\operatorname{PSp}_{4}\left(\mathbf{F}_{3}\right)$.

The case of a surjective representation $\bar{\rho}$ is of special interest, since this is what happens generically for the three-torsion Galois representations of abelian surfaces.

Theorem 2. Suppose that $\bar{\rho}$ is surjective. Then $\mathcal{A}_{2}(\bar{\rho})$ is not rational over $\mathbf{Q}$, and the minimal degree of any rational cover is 6 .

In light of the result BCGP18, Prop 10.2.3] mentioned above, the constant 6 is best possible in this case.

The key ingredient in our results is the explicit description of the cohomology of the compactified Siegel modular variety $\mathcal{A}_{2}^{*}(3)$ given in HW01. We use it to study the Galois module $\operatorname{Pic}_{\overline{\mathbf{Q}}}\left(\mathcal{A}_{2}^{*}(\bar{\rho})\right)$. The Galois action over $E=\mathbf{Q}(\sqrt{-3})$ factors through the projectivization of $\bar{\rho}$ turning it into a $H$-module. We then calculate group cohomology of this module for various subgroups $P \subset H$, and employ a necessary criterion for rationality (see Theorem 3) to deduce our results.

1.1. Acknowledgments. We thank Jason Starr and Yuri Tschinkel for discussions about rationality versus geometric rationality for smooth varieties over number fields, Steven Weintraub for a suggestion on how to explicitly extract a description of $H^{2}\left(\mathcal{A}_{2}^{*}(3), \mathbf{Z}\right)$ as a $G=\operatorname{PSp}_{4}\left(\mathbf{F}_{3}\right)$-module from Theorem 4.9 of [HW01, and Mark Watkins with help using Magma BCP97. We thank the anonymous referees for useful comments and corrections, and we also thank Nils Bruin for explaining to us many of the ideas in section 2.3 .

\section{StRATEGy}

The main idea behind the proof is to follow a strategy employed by Manin for cubic surfaces. Recall [Man86, §A.1] that a continuous $G_{K}$-module with the discrete topology is called a permutation module if it admits a finite free $\mathbf{Z}$-basis on which $G_{K}$ acts (via a finite quotient) via permutations, and that two $G_{K^{-}}$ modules $M$ and $N$ are similar if $M \oplus P \simeq N \oplus Q$ for some permutation modules $P$ and $Q$. In particular, we employ the following theorem.

Theorem 3. Man86, §A.1 Theorem 2] Let $Z$ be a smooth projective algebraic variety over a number field $K$. Suppose that $Z$ is rational over $K$. Then $\operatorname{Pic}_{\bar{K}} Z$ 
as a $G_{K}$-module is stably permutation. In other words, it is similar to the zero module.

The Shimura variety $\mathcal{A}_{2}(3)$ admits a smooth toroidal projective compactification $\mathcal{A}_{2}^{*}(3)$, the (canonical) toroidal compactification constructed by Igusa Igu67. The automorphism group of $\mathcal{A}_{2}^{*}(3)$ over $\overline{\mathbf{Q}}$ is the group $G=\operatorname{PSp}_{4}\left(\mathbf{F}_{3}\right)$, the simple group of order 25920 , which acts over the field $E=\mathbf{Q}(\sqrt{-3})$. It will be convenient from this point onwards to always work over the field $E$. (Certainly rationality over $\mathbf{Q}$ implies rationality over $E$, so non-rationality over $E$ implies non-rationality over Q.) This action on $\mathcal{A}_{2}(3)$ arises explicitly from the action of $G$ on the 3torsion $A[3]=(\mathbf{Z} / 3 \mathbf{Z})^{2} \oplus\left(\mu_{3}\right)^{2} \simeq(\mathbf{Z} / 3 \mathbf{Z})^{4}$ over $E$. We will apply Theorem 3 to the corresponding twist $\mathcal{A}_{2}^{*}(\bar{\rho})$. We then make crucial use of very explicit description of the cohomology of this compactification given by Hoffman and Weintraub [HW01]. We recall some facts from that paper here now.

2.1. Picard group. The Picard group of $\mathcal{A}_{2}^{*}(3)$ over $\overline{\mathbf{Q}}$ is a free $\mathbf{Z}$-module of rank 61. It is generated by two natural sets of classes. The first is a 40-dimensional space explained by the 40 connected components of the boundary. The second is a 45-dimensional space explained by divisors coming from Humbert surfaces. These are also in one to one correspondence with the 45 nodes on the Burkhardt quartic. Together, these generate the Picard group of $\mathcal{A}_{2}^{*}(3)$ over $\overline{\mathbf{Q}}$, which is free of rank 61 . Indeed, the Betti cohomology of $\mathcal{A}_{2}^{*}(3)$ over $\mathbf{Z}$ is free of degrees $1,0,61,0,61,0,1$ for $i=0, \ldots 6$ by [HW01, Theorem 1.1]. Furthermore, all of these classes are trivial under the action of $G_{E}$.

Let $\bar{\rho}: G_{\mathbf{Q}} \rightarrow \mathrm{GSp}_{4}\left(\mathbf{F}_{3}\right)$ be a continuous Galois representation with cyclotomic similitude character. The assumption on the similitude character implies that the restriction of $\bar{\rho}$ to $E$ is valued in $\operatorname{Sp}_{4}\left(\mathbf{F}_{3}\right)$. Let

$$
\varrho: G_{E} \rightarrow G=\operatorname{PSp}_{4}\left(\mathbf{F}_{3}\right)
$$

denote the projectivization of the representation $\bar{\rho}$ restricted to $E$. The group $G$ acts over $E$ on $\mathcal{A}_{2}^{*}(3)$ via automorphisms, and $\mathcal{A}_{2}^{*}(\bar{\rho})$ is the twist of $\mathcal{A}_{2}^{*}(3)$ by $\varrho$. The group $\operatorname{Pic}_{\overline{\mathbf{Q}}} \mathcal{A}_{2}^{*}(\bar{\rho})$ as a $G_{E}$-module is obtained by considering $\operatorname{Pic} \overline{\mathbf{Q}} \mathcal{A}_{2}^{*}(3)$ as a $G$-module and then obtaining the Galois action via the map $\varrho: G_{E} \rightarrow G$. Thus it remains to closely examine $\operatorname{Pic} \overline{\mathbf{Q}}\left(\mathcal{A}_{2}^{*}(3)\right)$ as a $G$-module over $\mathbf{Z}$. In fact, we can quickly prove a weaker version of Theorem 2 by studying this $G$-module over $\mathbf{Q}$. The group $G$ admits a unique conjugacy class $G_{45}$ of subgroups of index 45 , but two conjugacy classes of index 40; let $G_{40}$ denote the (conjugacy class of) subgroups which fix a point in the tautological action of $G \subset \mathrm{PGL}_{4}\left(\mathbf{F}_{3}\right)$ on $\mathbf{P}^{3}\left(\mathbf{F}_{3}\right)$. The following is an easy consequence of the calculations of HW01. (and is also confirmed by our Magma code).

Lemma 1. As $\mathbf{Q}[G]$-modules, there is an equality of virtual representations

$$
H^{2}\left(\mathcal{A}_{2}^{*}(3), \mathbf{Q}\right) \simeq \operatorname{Pic}_{\overline{\mathbf{Q}}}\left(\mathcal{A}_{2}^{*}(3)\right) \otimes \mathbf{Q}=\mathbf{Q}\left[G / G_{40}\right]+\mathbf{Q}\left[G / G_{45}\right]-\left[\chi_{24}\right],
$$

where $\chi_{24} \otimes_{\mathbf{Q}} \mathbf{C}$ is the unique absolutely irreducible 24-dimensional representation of $G$.

Now, assuming that $\varrho$ is surjective, we can prove that $\mathcal{A}_{2}^{*}(\bar{\rho})$ is not rational simply by proving that $\chi_{24}$ is not virtually equal to a sum of permutation representations. If $R_{\mathbf{Q}}(G)$ denotes the representation $\operatorname{ring}$ of $G$, this is equivalent to 
proving that $\chi_{24} \in R_{\mathbf{Q}}(G)$ does not lie in the Burnside subring generated by permutation representations. But one may compute (using Magma or otherwise) that the Burnside cokernel of $G$ has order 2 and is generated by $\chi_{24}$. This proves a weaker version of Theorem 2 showing that any rational cover of $\mathcal{A}_{2}(\bar{\rho})$ should have degree at least 2 , although it is softer in that it only needs the $\mathbf{Q}[G]$-representation rather than the $\mathbf{Z}[G]$-module. This argument also applies if one only assumes that the image of $\varrho$ is $H \subset G$, as long as the restriction of $\chi_{24}$ to $H$ is still non-trivial in the Burnside cokernel, which it is for precisely 8 of the 116 conjugacy classes of subgroups of $G$.

2.2. Cohomological Obstructions. From now on, we let $H$ denote the image of $\varrho: G_{E} \rightarrow G=\mathrm{PSp}_{4}\left(\mathbf{F}_{3}\right)$. A second way to prove that a Galois module is not similar to the zero module is to use cohomology. If $M$ is a permutation module of $H$, then the restriction of $M$ to any subgroup $P$ is also a permutation module, and thus a direct sum of $P$-modules of the form $\mathbf{Z}[P / Q]$ for subgroups $Q$ of $P$. (Note that since a permutation module of a group $G$ arises from a finite $G$-set, it always decomposes over $\mathbf{Z}$ into a direct sum of such irreducible permutation modules.) Then, Shapiro's Lemma implies that $H^{1}(P, M)$ is a direct sum of groups of the form

$$
H^{1}(P, \mathbf{Z}[P / Q])=H^{1}(Q, \mathbf{Z})=0,
$$

where the second group vanishes because $Q$ is finite. Moreover, the $\mathbf{Z}$-dual $M^{\vee}=$ $\operatorname{Hom}(M, \mathbf{Z})$ of a permutation module is isomorphic to the same permutation module (a permutation matrix is its own inverse transpose). Thus one immediately has the following elementary criterion.

Lemma 2 (Cohomological Criterion for non-rationality). Let $M$ denote the $G$ module $\operatorname{Pic}_{\overline{\mathbf{Q}}}\left(\mathcal{A}_{2}^{*}(3)\right)$. Suppose $\mathcal{A}_{2}^{*}(\bar{\rho})$ is rational over $E=\mathbf{Q}(\sqrt{-3})$, and $\left.\varrho\right|_{G_{E}}$ has image $H \subset G$. Then

$$
H^{1}\left(P, M^{\vee}\right)=H^{1}(P, M)=0
$$

for every subgroup $P \subset H$.

We note that this is not an "if and only if" criterion. In the language of [CTS77, the lemma is saying that $M$ as a $G_{E}$-module is flasque and coflasque respectively. In general, this is weaker than being stably permutation (which itself is not enough to formally imply rationality).

In order to test this criterion in practice, we need an explicit description of $M$ as a $\mathbf{Z}[G]$-module rather than a $\mathbf{Q}[G]$-module. In order to do this, we explain how an explicit description of $M$ can be extracted from Theorem 4.9 of HW01. That theorem describes a set of elements which generate both $H_{4}\left(\mathcal{A}_{2}^{*}(3), \mathbf{Z}\right)$ and $H^{2}\left(\mathcal{A}_{2}^{*}(3), \mathbf{Z}\right)$, and explicitly gives the intersection pairing between them. Moreover, the basis comes with a transparent action of the group $G$. Specifically, $H^{2}\left(\mathcal{A}_{2}^{*}(3), \mathbf{Z}\right)$ is given as a quotient of $\mathbf{Z}\left[G / G_{40}\right] \oplus \mathbf{Z}\left[G / G_{45}\right]$. Hence to compute $H^{2}\left(\mathcal{A}_{2}^{*}(3), \mathbf{Z}\right)$ as a $G$ module, it suffices to compute the quotient of $\mathbf{Z}\left[G / G_{40}\right] \oplus \mathbf{Z}\left[G / G_{45}\right]$ by the saturated subspace which pairs trivially with all elements of $H_{4}\left(\mathcal{A}_{2}^{*}(3), \mathbf{Z}\right)$. Having carried out this computation, we obtain a free abelian group of rank 61 with an explicit action of $G$. We then do the following for every conjugacy class of subgroups $H \subset G$.

(1) Determine whether $\chi_{24}$ is non-trivial in the Burnside cokernel of $H$.

(2) Determine whether $H^{1}(P, M) \neq 0$ for any subgroup $P \subset H$.

(3) Determine whether $H^{1}\left(P, M^{\vee}\right) \neq 0$ for any subgroup $P \subset H$. 
If any of these is non-trivial, this proves that $\mathcal{A}_{2}^{*}(\bar{\rho})$ is not rational. Moreover, the computation of these cohomology groups allows us to deduce our result about the minimal degree of any rational covering.

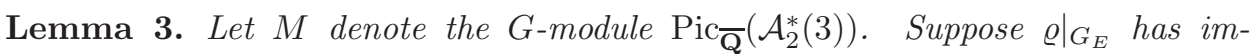
age $H \subset G$. Let $n$ denote the least common multiple of the exponents of $H^{1}(P, M)$ and $H^{1}\left(P, M^{\vee}\right)$ as $P$ varies over all subgroups of $H$. Suppose $f: X \rightarrow \mathcal{A}_{2}^{*}(\bar{\rho})$ is a rational cover of degree $d$ defined over $\mathbf{Q}$. Then $n$ divides $d$.

Proof. The induced pullback map $f^{*}: \operatorname{Pic}_{\overline{\mathbf{Q}}}\left(\mathcal{A}_{2}^{*}(\bar{\rho})\right) \rightarrow \operatorname{Pic}_{\overline{\mathbf{Q}}}(X)$ and pushforward $\operatorname{map} f_{*}: \operatorname{Pic} \overline{\mathbf{Q}}(X) \rightarrow \operatorname{Pic}_{\overline{\mathbf{Q}}}\left(\mathcal{A}_{2}^{*}(\bar{\rho})\right)$ are Galois equivariant since $f$ is defined over $\mathbf{Q}$.

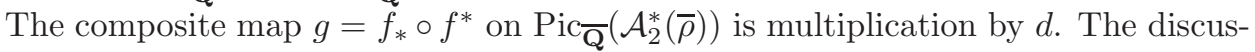
sion in $\$ 2.1$ shows that the $G_{E}$-module $\operatorname{Pic} \overline{\mathbf{Q}}\left(\mathcal{A}_{2}^{*}(\bar{\rho})\right)$ can be thought of as the $H$ module $M$.

By Theorem 3, we know that $\operatorname{Pic}_{\overline{\mathbf{Q}}}(X)$ is stably permutation as a Galois module and hence the Galois cohomology group $H^{1}\left(G_{\mathbf{Q}}, \operatorname{Pic}_{\overline{\mathbf{Q}}}(X)\right)=0$. Therefore, the maps induced by $g$ on the cohomology groups $H^{1}(P, M)$ and $H^{1}\left(P, M^{\vee}\right)$ are the zero maps for every subgroup $P \subset H$. Since the map $g$ is multiplication by $d$, the induced map on cohomology is also multiplication by $d$, and hence we deduce that the exponent of each of these cohomology groups divides $d$.

We give one final statement which can be extracted from Magma using the code given in [CC21, but not directly from the table. In order to represent elements of $G=\operatorname{PSp}_{4}\left(\mathbf{F}_{3}\right)$ by matrices, we follow the conventions of Magma by fixing $\operatorname{Sp}_{4}\left(\mathbf{F}_{3}\right) \subset \mathrm{GL}_{4}\left(\mathbf{F}_{3}\right)$ to be the matrices preserving the symplectic form

$$
J=\left(\begin{array}{cccc}
0 & 0 & 0 & 1 \\
0 & 0 & 1 & 0 \\
0 & -1 & 0 & 0 \\
-1 & 0 & 0 & 0
\end{array}\right)
$$

Lemma 4. Suppose that the image of $\bar{\rho}$ contains an element conjugate in $\operatorname{PSp}_{4}\left(\mathbf{F}_{3}\right)$ to

$$
\left(\begin{array}{cccc}
1 & 0 & 0 & -1 \\
0 & 1 & 1 & 0 \\
0 & 0 & 1 & 0 \\
0 & 0 & 0 & 1
\end{array}\right) .
$$

Then $\mathcal{A}_{2}(\bar{\rho})$ is not rational, and the minimal degree of any rational cover is divisible by 3 .

Proof. It suffices to note that this element generates the subgroup labelled as subgroup 6 in the table below, and then to apply Lemma 3 .

2.3. Other cases where rationality can be established. The analysis of Baker's parametrization Bak46] undertaken in [BN18, §4] allows one to deduce the rationality of certain twists of the Burkhardt quartic $B$ (and hence of $\mathcal{A}_{2}(\bar{\rho})$ ) in a few more cases. (We thank Nils Bruin for pointing this out to us, as well as explaining the geometric construction below.) The rational parametrization $\mathbf{P}^{3} \rightarrow B$ over $\mathbf{Q}$ constructed in [BN18] is not equivariant with respect the action of $\operatorname{PSp}_{4}\left(\mathbf{F}_{3}\right)$. If it were, then the twists $\mathcal{A}_{2}(\bar{\rho})$ we are considering would all be birational to BrauerSeveri varieties. However, because they are also unirational over $\mathbf{Q}$ by BCGP18, Prop 10.2.3], they would be rational over $\mathbf{Q}$, which we prove in this paper to be 
false in general. On the other hand, the parametrization $\mathbf{P}^{3} \rightarrow B$ is equivariant with respect to the (unique up to conjugacy) cyclic group of order 9 BN18, $\S 4.3]$, and also with respect to the corresponding group scheme over $\mathbf{Q}$ whose $E$ points are this group of order 9 (c.f. [CCR20, §2.3]), which controls the descent from $E$ to $\mathbf{Q}$. In particular, the same argument implies that $\mathcal{A}_{2}(\bar{\rho})$ is rational in two further cases, namely, the subgroups labelled $n=4$ (of order 3 ) and $n=24$ (of order 9) in the table below. One can also arrive at this rational parametrization more geometrically, following [BN18, §4], whose notation we now freely follow. The variety of lines $L_{J_{1}, J_{2}, J_{3}}$ incident with 3 -distinct planes $J_{i} \subset \mathbf{P}^{4}$ is geometrically rational. If these planes are mutually skew and lie on $B$, there is a dominant map $L_{J_{1}, J_{2}, J_{3}} \rightarrow B$ defined by noting that a line will generically intersect $B$ in four points and each $J_{i}$ in one point, and hence one can send the line to the fourth point of intersection with $B$. There are 40 Jacobi planes $J_{i}$ on $B$, and 2880 triples of mutually skew such planes. The stabilizer under $\operatorname{PSp}_{4}\left(\mathbf{F}_{3}\right)$ on these 2880 triples is the cyclic group of order 9 . The assumption that $H$ is contained inside this group then implies that there exists a triple of $\operatorname{Gal}(\overline{\mathbf{Q}} / \mathbf{Q})$-invariant mutually skew planes on the twist of $B$ corresponding to $\bar{\rho}$. The result then follows after noting that $L_{J_{1}, J_{2}, J_{3}}$ is rational over $\mathbf{Q}$ whenever this triple is defined over $\mathbf{Q}$. (We omit a direct proof of this last claim in light of the alternate argument given above.)

\section{Computation}

Let $M$ denote the $G$-module $\operatorname{Pic}_{\overline{\mathbf{Q}}}\left(\mathcal{A}_{2}^{*}(3)\right) \simeq H^{2}\left(\mathcal{A}_{2}^{*}(3), \mathbf{Z}\right)$. We have, by Poincaré duality, an isomorphism $M^{\vee}=H^{4}\left(\mathcal{A}_{2}^{*}(3), \mathbf{Z}\right)$. Below we present in a table the result of our computation for all 116 conjugacy classes of subgroups $H \subset G$, indicating the following data:

(1) An ordering $n=1 \ldots 116$ of the conjugacy class of the subgroup $H$ as determined by Magma.

(2) The group $H$ in the small groups database BEO01. The first element of the pair gives the order of $H$.

(3) The order of $M$ in the Burnside cokernel of $H$ over $\mathbf{Q}$ (if it is non-trivial). If this is greater than 1, then the corresponding twist is not rational over $E$ (or $\mathbf{Q}$ ).

(4) The least common multiple of the exponents of $H^{1}(P, M)$ and $H^{1}\left(P, M^{\vee}\right)$ as $P$ ranges over subgroups $P \subset H$. If this is greater than 1 , then the corresponding twist is not rational over $E$ (or $\mathbf{Q}$ ). In particular, the fact that this number is 6 for $G$ itself proves Theorem 2,

(5) The pre-image of $H$ in $\operatorname{Sp}_{4}\left(\mathbf{F}_{3}\right)$ acts on $\mathbf{F}_{3}^{4}$. Is this action absolutely irreducible? (That is, is the action on $\overline{\mathbf{F}}_{3}^{4}$ irreducible.)

(6) A list of the conjugacy class of maximal subgroups of $H$ (as indexed in the table). This allows one to compute the LCM column directly. The table is separated into blocks to reflect the geometry of the corresponding poset of subgroups. In particular, all maximal subgroups of $H$ occur in blocks before that of $H$.

(7) The last two columns give $H^{1}(H, M)$ and $H^{1}\left(H, M^{\vee}\right)$.

One must be careful while reading the table because the ordering of the conjugacy classes of subgroups is not canonical. The Small Group tag and the indices of the maximal subgroups given in the second and sixth columns of the table do, however, 
determine the ordering uniquely once we distinguish between the conjugacy classes indexed by $n=2,3, n=4,5,6, n=9,11$, and $n=10,12$. This can be done by considering the length of each of these conjugacy classes (i.e., the number of subgroups in each conjugacy class) as shown in the following table.

\begin{tabular}{|c|c|}
\hline$n$ & Length \\
\hline 2 & 45 \\
3 & 270 \\
\hline 4 & 40 \\
5 & 120 \\
6 & 240 \\
\hline
\end{tabular}

\begin{tabular}{|c|c|}
\hline$n$ & Length \\
\hline 9 & 270 \\
11 & 405 \\
\hline 10 & 270 \\
12 & 540 \\
\hline
\end{tabular}

The Magma code available at CC21 computes $G$ and $M$ directly from the description given by Hoffman and Weintraub HW01. This leads to a representation of $G$ as generated by two sparse $61 \times 61$ matrices $x$ and $y$ in $\mathrm{GL}_{61}(\mathbf{Z})$ such that the underlying module on which $G$ acts (on the right, by Magma conventions) is $M$. The matrices $x$ and $y$ are also printed in the output file of our Magma script.

\begin{tabular}{|c|c|c|c|c|c|c|c|}
\hline$n$ & SmallGroup & $\mathrm{B}$ & LCM & irred & maximal subgroups & $H^{1}(M)$ & $H^{1}\left(M^{\vee}\right)$ \\
\hline 1 & $\langle 1,1\rangle$ & & 1 & no & & & \\
\hline 2 & $\langle 2,1\rangle$ & & 1 & no & 1 & & \\
\hline 3 & $\langle 2,1\rangle$ & & 1 & no & 1 & & \\
\hline 4 & $\langle 3,1\rangle$ & & 1 & no & 1 & & \\
\hline 5 & $\langle 3,1\rangle$ & & 1 & no & 1 & & \\
\hline 6 & $\langle 3,1\rangle$ & & 3 & no & 1 & $\mathbf{Z} / 3 \mathbf{Z}$ & $\mathbf{Z} / 3 \mathbf{Z}$ \\
\hline 7 & $\langle 5,1\rangle$ & & 1 & no & 1 & & \\
\hline 8 & $\langle 4,1\rangle$ & & 1 & no & 2 & & \\
\hline 9 & $\langle 4,2\rangle$ & & 1 & no & 23 & & \\
\hline 10 & $\langle 4,2\rangle$ & & 2 & no & 3 & & $(\mathbf{Z} / 2 \mathbf{Z})^{2}$ \\
\hline 11 & $\langle 4,2\rangle$ & & 2 & no & 23 & & $\mathbf{Z} / 2 \mathbf{Z}$ \\
\hline 12 & $\langle 4,2\rangle$ & & 1 & no & 3 & & \\
\hline 13 & $\langle 4,1\rangle$ & & 1 & no & 3 & & \\
\hline 14 & $\langle 6,1\rangle$ & & 3 & no & 26 & $\mathbf{Z} / 3 \mathbf{Z}$ & \\
\hline 15 & $\langle 6,2\rangle$ & & 1 & no & 24 & & \\
\hline 16 & $\langle 6,2\rangle$ & & 3 & no & 26 & & \\
\hline 17 & $\langle 6,1\rangle$ & & 3 & no & 36 & & $\mathrm{Z} / 3 \mathrm{Z}$ \\
\hline 18 & $\langle 6,1\rangle$ & & 1 & no & 35 & & \\
\hline 19 & $\langle 6,2\rangle$ & & 1 & no & 25 & & \\
\hline 20 & $\langle 6,2\rangle$ & & 1 & no & 35 & & \\
\hline 21 & $\langle 9,2\rangle$ & & 3 & no & 56 & & $(\mathbf{Z} / 3 \mathbf{Z})^{2}$ \\
\hline 22 & $\langle 9,2\rangle$ & & 3 & no & 46 & & $(\mathbf{Z} / 3 \mathbf{Z})^{2}$ \\
\hline 23 & $\langle 9,2\rangle$ & & 3 & no & 456 & & \\
\hline 24 & $\langle 9,1\rangle$ & & 1 & no & 4 & & \\
\hline 25 & $\langle 10,1\rangle$ & & 1 & no & 37 & & \\
\hline 26 & $\langle 8,4\rangle$ & & 1 & no & 8 & & \\
\hline 27 & $\langle 8,5\rangle$ & & 2 & no & 1112 & & $(\mathbf{Z} / 2 \mathbf{Z})^{2}$ \\
\hline 28 & $\langle 8,5\rangle$ & & 2 & no & 1011 & & $(\mathbf{Z} / 2 \mathbf{Z})^{2}$ \\
\hline 29 & $\langle 8,5\rangle$ & & 2 & no & 91011 & & \\
\hline 30 & $\langle 8,2\rangle$ & & 2 & no & 811 & & \\
\hline 31 & $\langle 8,2\rangle$ & & 2 & no & 1113 & $\mathbf{Z} / 2 \mathbf{Z}$ & $\mathbf{Z} / 2 \mathbf{Z}$ \\
\hline
\end{tabular}




\begin{tabular}{|c|c|c|c|c|c|c|c|}
\hline \multicolumn{8}{|c|}{ F. CALEGARI AND S. CHIDAMBARAM } \\
\hline 32 & $\langle 8,3\rangle$ & & 2 & no & 811 & \multirow{21}{*}{$\mathbf{Z} / 3 \mathbf{Z}$} & $\mathbf{Z} / 2 \mathbf{Z}$ \\
\hline 33 & $\langle 8,3\rangle$ & & 2 & no & 101213 & & $\mathbf{Z} / 2 \mathbf{Z}$ \\
\hline 34 & $\langle 8,3\rangle$ & & 1 & no & 91213 & & \\
\hline 35 & $\langle 12,3\rangle$ & & 2 & no & 510 & & \\
\hline 36 & $\langle 12,3\rangle$ & & 3 & no & 612 & & $\mathbf{Z} / 3 \mathbf{Z}$ \\
\hline 37 & $<12,4>$ & & 3 & no & 9141617 & & \\
\hline 38 & $<12,5\rangle$ & & 1 & no & 91920 & & \\
\hline 39 & $<12,1>$ & & 1 & no & 1320 & & \\
\hline 40 & $<12,2\rangle$ & & 1 & no & 815 & & \\
\hline 41 & $<12,4>$ & & 1 & no & 121820 & & \\
\hline 42 & $\langle 18,4\rangle$ & & 3 & no & 171821 & & $(\mathbf{Z} / 3 \mathbf{Z})^{2}$ \\
\hline 43 & $\langle 18,3\rangle$ & & 3 & no & 141621 & & \\
\hline 44 & $\langle 18,3\rangle$ & & 3 & no & 141923 & & \\
\hline 45 & $\langle 18,3\rangle$ & & 3 & no & 141522 & & \\
\hline 46 & $\langle 18,3\rangle$ & & 3 & no & 182021 & & $\mathbf{Z} / 3 \mathbf{Z}$ \\
\hline 47 & $\langle 18,3\rangle$ & & 3 & no & 172023 & & \\
\hline 48 & $\langle 18,5\rangle$ & & 3 & no & 15161923 & & \\
\hline 49 & $<20,3\rangle$ & & 1 & yes & 1325 & & \\
\hline 50 & $\langle 27,5\rangle$ & & 3 & no & 212223 & & $\mathbf{Z} / 3 \mathbf{Z}$ \\
\hline 51 & $<27,3\rangle$ & & 3 & no & 22 & & $(\mathbf{Z} / 3 \mathbf{Z})^{2}$ \\
\hline 52 & $\langle 27,4\rangle$ & & 3 & no & 2224 & & $\mathbf{Z} / 3 \mathbf{Z}$ \\
\hline 53 & $\langle 16,14\rangle$ & & 2 & yes & 2829 & \multirow{27}{*}{$\mathbf{Z} / 3 \mathbf{Z}$} & \\
\hline 54 & $\langle 16,13\rangle$ & & 2 & no & 263032 & & \\
\hline 55 & $\langle 16,11\rangle$ & & 2 & yes & 27283032 & & $\mathbf{Z} / 2 \mathbf{Z}$ \\
\hline 56 & $\langle 16,3\rangle$ & & 2 & no & 2831 & & $(\mathbf{Z} / 2 \mathbf{Z})^{2}$ \\
\hline 57 & $\langle 16,11\rangle$ & & 2 & yes & 2729313334 & & $\mathbf{Z} / 2 \mathbf{Z}$ \\
\hline 58 & $\langle 16,3\rangle$ & & 2 & no & 293031 & & \\
\hline 59 & $\langle 24,3\rangle$ & & 1 & no & 1526 & & \\
\hline 60 & $\langle 24,13\rangle$ & & 2 & no & 202935 & & \\
\hline 61 & $\langle 24,3\rangle$ & & 3 & no & 1626 & & \\
\hline 62 & $\langle 24,3\rangle$ & & 1 & no & 1926 & & \\
\hline 63 & $\langle 24,11\rangle$ & 2 & 1 & no & 2640 & & \\
\hline 64 & $\langle 24,13\rangle$ & & 2 & no & 192835 & & \\
\hline 65 & $\langle 24,13\rangle$ & & 6 & no & 162736 & & \\
\hline 66 & $\langle 24,12\rangle$ & & 2 & no & 183335 & & \\
\hline 67 & $\langle 24,12\rangle$ & & 6 & no & 173336 & & $\mathbf{Z} / 6 \mathbf{Z}$ \\
\hline 68 & $\langle 24,12\rangle$ & & 3 & no & 143436 & & \\
\hline 69 & $<24,8>$ & & 1 & no & 34383941 & & \\
\hline 70 & $\langle 36,10\rangle$ & & 3 & no & 374243 & & \\
\hline 71 & $\langle 36,10\rangle$ & & 3 & no & 414246 & & $\mathbf{Z} / 3 \mathbf{Z}$ \\
\hline 72 & $\langle 36,9\rangle$ & & 3 & no & 1342 & & $\mathbf{Z} / 3 \mathbf{Z}$ \\
\hline 73 & $\langle 36,12\rangle$ & & 3 & no & 3738444748 & & \\
\hline 74 & $<54,8>$ & & 3 & no & 4551 & & \\
\hline 75 & $\langle 54,13\rangle$ & & 3 & no & 42464750 & & $\mathbf{Z} / 3 \mathbf{Z}$ \\
\hline 76 & $<54,12\rangle$ & & 3 & no & 4344454850 & & \\
\hline 77 & $\langle 60,5\rangle$ & & 2 & no & 182535 & & \\
\hline 78 & $\langle 60,5\rangle$ & & 3 & no & 172536 & & $\mathbf{Z} / 3 \mathbf{Z}$ \\
\hline 79 & $<81,7\rangle$ & & 3 & no & 505152 & & $\mathbf{Z} / 3 \mathbf{Z}$ \\
\hline
\end{tabular}




\begin{tabular}{|c|c|c|c|c|c|c|}
\hline 80 & $\langle 32,49\rangle$ & & 2 & no & 5456 & \\
\hline 81 & $\langle 32,6\rangle$ & & 2 & yes & 5556 & $\mathbf{Z} / 2 \mathbf{Z}$ \\
\hline 82 & $<32,27\rangle$ & & 2 & yes & 5355565758 & \\
\hline 83 & $\langle 48,30\rangle$ & & 2 & no & 395860 & \\
\hline 84 & $<48,49\rangle$ & & 2 & yes & 38536064 & \\
\hline 85 & $\langle 48,33\rangle$ & & 2 & yes & 405459 & \\
\hline 86 & $\langle 48,48\rangle$ & & 2 & no & 41576066 & $\mathbf{Z} / 2 \mathbf{Z}$ \\
\hline 87 & $\langle 48,48\rangle$ & & 6 & yes & 3757656768 & \\
\hline 88 & $<72,40\rangle$ & & 3 & no & 34707172 & \\
\hline 89 & $\langle 72,25\rangle$ & 2 & 3 & no & 4859616263 & \\
\hline 90 & $<80,49>$ & & 2 & yes & 753 & \\
\hline 91 & $\langle 108,40\rangle$ & & 3 & no & 7175 & $\mathbf{Z} / 3 \mathbf{Z}$ \\
\hline 92 & $\langle 108,15\rangle$ & & 3 & no & 4074 & \\
\hline 93 & $\langle 108,38\rangle$ & & 3 & no & 70737576 & \\
\hline 94 & $<108,37\rangle$ & & 3 & no & 397275 & \\
\hline 95 & $<120,34>$ & & 3 & yes & 37496878 & \\
\hline 96 & $<120,34>$ & & 2 & yes & 41496677 & \\
\hline 97 & $\langle 162,10\rangle$ & & 3 & no & 747679 & \\
\hline 98 & $\langle 64,138\rangle$ & & 2 & yes & 808182 & \\
\hline 99 & $<96,204\rangle$ & & 2 & no & 626480 & \\
\hline 100 & $<96,204\rangle$ & & 6 & no & 616580 & \\
\hline 101 & $<96,201\rangle$ & 2 & 2 & no & 638085 & \\
\hline 102 & $\langle 96,195\rangle$ & & 2 & yes & 6982838486 & \\
\hline 103 & $<160,234\rangle$ & & 2 & yes & 258290 & \\
\hline 104 & $<216,88\rangle$ & 2 & 3 & no & 6392 & \\
\hline 105 & $\langle 216,158\rangle$ & & 3 & no & 6988919394 & \\
\hline 106 & $\langle 324,160\rangle$ & & 3 & no & 367991 & $\mathbf{Z} / 3 \mathbf{Z}$ \\
\hline 107 & $\langle 360,118\rangle$ & & 6 & no & 6667727778 & $\mathbf{Z} / 3 \mathbf{Z}$ \\
\hline 108 & $\langle 192,1493\rangle$ & & 6 & yes & 8798100 & \\
\hline 109 & $<192,201\rangle$ & & 2 & yes & 849899 & \\
\hline 110 & $<288,860\rangle$ & 2 & 6 & no & 8999100101 & \\
\hline 111 & $\langle 648,533\rangle$ & 2 & 3 & no & 8997104 & \\
\hline 112 & $<648,704>$ & & 3 & no & 6897105106 & \\
\hline 113 & $\langle 720,763\rangle$ & & 6 & yes & 8687889596107 & \\
\hline 114 & $\langle 576,8277\rangle$ & 2 & 6 & yes & 73108109110 & \\
\hline 115 & $\langle 960,11358\rangle$ & & 2 & yes & 77102103109 & \\
\hline 116 & $G$ & 2 & 6 & yes & 111112113114115 & \\
\hline
\end{tabular}

\section{REFERENCES}

[Bak46] H. F. Baker. A Locus with 25920 Linear Self-Transformations. Cambridge Tracts in Mathematics and Mathematical Physics, no. 39. Cambridge, at the University Press; New York, The Macmillan Company, 1946.

[BCGP18] George Boxer, Frank Calegari, Toby Gee, and Vincent Pilloni. Abelian surfaces over totally real fields are potentially modular. preprint, 2018.

[BCP97] Wieb Bosma, John Cannon, and Catherine Playoust. The Magma algebra system. I. The user language. J. Symbolic Comput., 24(3-4):235-265, 1997. Computational algebra and number theory (London, 1993).

[BEO01] Hans Ulrich Besche, Bettina Eick, and E. A. O'Brien. The groups of order at most 2000. Electron. Res. Announc. Amer. Math. Soc., 7:1-4, 2001. 
[BN18] Nils Bruin and Brett Nasserden. Arithmetic aspects of the Burkhardt quartic threefold. J. Lond. Math. Soc. (2), 98(3):536-556, 2018.

[CC21] Frank Calegari and Shiva Chidambaram. Auxiliary magma files. https://github.com/shiva-chid/code_rationality 2021.

[CCR20] Frank Calegari, Shiva Chidambaram, and David P. Roberts. Abelian surfaces with fixed 3-torsion. In Steven Galbraith, editor, Proceedings of the Fourteenth Algorithmic Number Theory Symposium (ANTS-XIV), Open Book Series 4, pages 91-108, Berkeley, 2020. Mathematical Sciences Publishers.

[CTS77] Jean-Louis Colliot-Thélène and Jean-Jacques Sansuc. La $R$-équivalence sur les tores. Ann. Sci. École Norm. Sup. (4), 10(2):175-229, 1977.

[HW01] J. William Hoffman and Steven H. Weintraub. The Siegel modular variety of degree two and level three. Trans. Amer. Math. Soc., 353(8):3267-3305, 2001.

[Igu67] Jun-Ichi Igusa. A desingularization problem in the theory of siegel modular functions. Mathematische Annalen, 168(1):228-260, Dec 1967.

[Man86] Yu. I. Manin. Cubic forms, volume 4 of North-Holland Mathematical Library. NorthHolland Publishing Co., Amsterdam, second edition, 1986. Algebra, geometry, arithmetic, Translated from the Russian by M. Hazewinkel.

Email address: fcale@uchicago.edu

The University of Chicago, 5734 S University Ave, Chicago, IL 60637, USA

Email address: shivac@mit.edu

Massachusetts Institute of Technology, 77 Massachusetts Ave. Cambridge, MA 02139, USA 